\title{
Aggression of Psychiatric Patients Associated with the Use of As-needed Medication
}

\author{
Authors \\ L. E. Goedhard ${ }^{1,2}$, J. J. Stolker ${ }^{1,2}$, H. L. I. Nijman ${ }^{3}$, A. C. G. Egberts ${ }^{2,4}$, E. R. Heerdink ${ }^{2}$ \\ Affiliations \\ Altrecht Institute for Mental Health Care, Den Dolder, The Netherlands \\ Department of Pharmacoepidemiology and Pharmacotherapy, Utrecht Institute for Pharmaceutical Sciences (UIPS), \\ Utrecht, The Netherlands \\ Faculty of Social Sciences, Radboud University Nijmegen, Nijmegen, The Netherlands \\ Department of Clinical Pharmacy, University Medical Center Utrecht, The Netherlands
}

\section{received $\quad 8.8 .2006$ \\ revised 17.11.2006 \\ accepted $\quad 15.12 .2006$}

Bibiliography

DOI 10.1055/s-2007-961817

Pharmacopsychiatry 2007;

40: 25-29

(c) Georg Thieme Verlag KG

Stuttgart · New York .

ISSN 0176-3679

Correspondence

E. R. Heerdink

Correspondence E. R. Heerdink Ph.D. Utrecht. Institute for Pharmaceutical Sciences. Department of Pharmacoepidemiology and Pharmacotherapy.P.O Box $80082 \cdot 3508$ TB Utrecht $\cdot$ The Netherlands Tel.: + 31/30/2537324

Fax: + 31/30/2539166 E.R.Heerdink@pharm.uu.nl

\section{Abstract \\ $\nabla$}

Introduction: Previous studies showed that aggression is an important reason to prescribe as-needed medication. The objective of this study was to compare the use of as-needed medication in aggressive and non-aggressive psychiatric patients and to explore patterns of administration of as-needed medication around aggressive incidents.

Methods: An observational study in three psychiatric wards was conducted. Incidence densities of as-needed medication were determined for aggressive and non-aggressive patients and expressed as incidence density ratios [IDRs]. Intensity of as-needed medication used before and following aggressive incidents were determined within a 48-hours time-window.

\section{Introduction}

Aggression by psychiatric patients has a large impact on well-being of patients and staff and is associated with high costs [5]. The incidence of aggressive incidents reported in different studies ranges from 0.4 to 59.9 incidents per patientyear, depending upon the type of ward and country in which the study was conducted [8]. To manage aggression, several intervention strategies are currently used, including pharmacotherapy, physical restraint and seclusion. Increased use of psychotropics by aggressive patients has been observed in observational studies. Higher dosages of psychotropics and polypharmacy are more common for aggressive patients than nonaggressive patients $[9,10]$. However, evidence for the effectiveness of pharmacological management of aggression is scarce [4]. Psychotropics generally are prescribed in a fixed dose regimen, but as-needed regimens for the management of aggression and aggression-related symptoms
Results: Aggressive patients had an increased use of both psychotropic and somatic as-needed medication (IDR, 2.5; 95\% CI, 2.2-2.7 and IDR, $2.1 ; 95 \% \mathrm{CI}, 1.8-2.4$, respectively). Of the psychotropic medication for aggressive patients, $15 \%$ was administered in a time-window of 48 hours around an aggressive incident; in this time-window more as-needed medication was administered following an aggressive incident compared to earlier treatments.

Conclusion: An increased use of both psychotropic and somatic as-needed medication is associated with aggressive behavior. Psychotropic as-needed medication is more frequently administered shortly after an aggressive incident than shortly before. However, more often as-needed medication is administered outside the 48 hours time-window around an aggressive incident. like agitation and disruptive behavior are also used $[2,3,6]$ giving "nursing staff greater freedom in administering medication, allowing them to administer rapidly in acute situations or at the patient's request" [12]. Especially antihistamines, antipsychotics and benzodiazepines are frequently used on an as-needed basis [3]. Although prescription of as-needed medication appears to be widespread in psychiatry, evidence for effectiveness is lacking $[11,12]$. We are unaware of clinical trials that specifically looked at the efficacy of as-needed regimens in adult psychiatry, and even descriptive and more exploratory studies about current practices of as-needed regimens are scarce.

Our study aims to investigate whether aggressive psychiatric patients have an increased use of asneeded medication, compared to non-aggressive patients. In addition, this study investigates the time-relationship between the occurrence of aggressive incidents and the administration of as-needed medication in this patient group. 


\section{Methods v \\ Setting, design and subjects}

A prospective observational study was conducted in three different psychiatric wards, located in the centre of the Netherlands. All patients, hospitalized for at least two weeks during the study-period of September 2004 until May 2005, were included. All the three participating wards were long-stay wards specialized in disruptive behavior, i.e., a forensic ward, a centre for patients with mild learning disabilities and disruptive behavior, and an orthopsychiatric ward.

The study protocol was approved by the Scientific Committee and the Board of the hospital. Patients were informed about the study and patients were not included in case of objection to participation in the study.

\section{Data collection}

Patient characteristics were collected from the hospital database. This database contains information about demographics, DSM-IV diagnoses at admission as established by psychiatrists of the ward, compulsory hospitalization and their history of earlier admissions. When information in the patient administration database was incomplete, additional data were collected from the medical records.

Aggressive behavior was assessed by using the Staff Observation Aggression Scale-Revised (SOAS-R), a validated aggression measurement instrument [7]. This scale measures the frequency, nature, and severity of both outwardly and inwardly directed aggressive behavior. SOAS-R scores range from 0 (no aggression) up to 22 (extremely severe aggression). For this study only outwardly directed aggressive incidents were analyzed, i.e., all forms of self-harming and suicidal behavior were excluded. Patients were categorized as aggressive if one or more aggressive incidents were recorded on the SOAS-R during the study period. Furthermore, aggressive patients were sub-classified as being mildly or severely aggressive. A patient was defined as being severely aggressive in case he or she was involved in one or more episodes of physical aggression towards person(s). In case a patient had exclusively displayed verbal aggression and/ or aggression towards objects he or she was categorized as being mildly aggressive. If two aggressive incidents of one patient occurred within one hour, they were regarded as one incident. As-needed medication was defined as medication which was not administered on a regular basis, but on the patient's or nurse's initiative. Hospital policy requires ward personnel to register all administrations of as-needed medications to every individual patient on standard forms. Patient name, drug name, date, time and dosage were registered. The pharmacy database was used to obtain additional information about the medication the patients received as part of their regular treatment regimens.

\section{Outcome}

Firstly, we aimed to investigate whether aggressive patients have an increased use of as-needed medication compared to non-aggressive patients. The primary outcome of this research question was the incidence density (ID) of psychotropic asneeded medication administrations for aggressive and nonaggressive patients. The ID was calculated by dividing the sum of administrated as-needed medications in one group by the total duration of follow-up in that group. The IDs for aggressive and non-aggressive patients were compared and expressed as an
Incidence Density Ratio (IDR). Selected psychotropic drugs included benzodiazepines, antipsychotics and promethazine. Secondary outcome was the ID of somatic as-needed medication administrations, e.g., analgesics like acetaminophen and ibuprofen.

The second aim of this study was to investigate the temporal association between the occurrence of aggressive incidents and the administration of psychotropic as-needed medication. Outcome for this research study was the intensity of psychotropic as-needed medication use - i.e., the number of as-needed medications in a defined interval of time divided by the total number of aggressive incidents - for different time-windows before and following aggressive incidents. To avoid overlap of the timewindows around consecutive aggressive incidents, only aggressive incidents with a preceding 24-hours incident free period were included.

\section{Analysis}

Between-group characteristics were analyzed using the chisquare for categorical variables and the Mann-Whitney U test for continuous, skewed variables.

For the comparison of the incidence densities between aggressive and non-aggressive patients, incidence density ratios (IDRs) and their $95 \% \mathrm{CI}$ were computed. To check the validity of this method, the use of aggressive patients compared to non-aggressive patients was also analyzed using a multiplicative intensity model [1], a generalization of cox proportional hazards regression model.

To investigate the temporal association between the administration of psychotropic as-needed medication and the occurrence of aggressive incidents, the frequencies of as-needed medication administrations (=intensity) before and following an aggressive incident were compared. A Poisson distribution was observed for the administration, which could be analyzed using a multiplicative intensity model. Differences in intensity of use before and following aggressive incidents were expressed as intensity ratios (IR). Data were analyzed using S-PLUS 6.

\section{Results \\ $\nabla$}

The initial study population was comprised of 130 patients. Three patients, however, objected to study participation and were therefore not included in the analysis. Furthermore, two patients who were hospitalized for less than two weeks were excluded. The mean follow-up was 170 days (range 14-273 days). Characteristics of the remaining 125 included patients are presented in 0 Table 1 . Aggressive patients $(n=76)$ turned out to differ significantly from non-aggressive patients $(n=49)$ in terms of age, ward, sex, and a diagnosis of "conduct disorder".

During the study period, 551 aggressive incidents caused by 76 patients, were recorded. Of these 76 patients, 61 were sub-classified as severely aggressive and 15 as mildly aggressive. Characteristics of the aggressive incidents are displayed in 0 Table 2. More, but less severe, incidents were recorded at the orthopsychiatric ward. At the centre for mildly mentally disabled patients a higher frequency and severity of aggressive incidents was observed compared to the two other wards.

In the nine-month of study period, 4427 as-needed medications were administered to 44 (60\%) aggressive and 20 (41\%) nonaggressive patients. The most frequently administered types of psychotropic medications $(n=2940)$ were benzodiazepines 
Table 1 Characteristics of the study population

\begin{tabular}{|c|c|c|c|c|c|}
\hline \multirow[t]{2}{*}{ Characteristics } & \multicolumn{2}{|c|}{$\begin{array}{l}\text { Patients with aggressive } \\
\text { incident(s) }(n=76)\end{array}$} & \multicolumn{2}{|c|}{$\begin{array}{l}\text { Patients without aggressive } \\
\text { incident(s) }(n=49)\end{array}$} & \multirow[t]{2}{*}{ p-Value } \\
\hline & No. & (\%) & No. & (\%) & \\
\hline Age (mean) & 27.2 years & - & 31.7 years & - & 0.004 \\
\hline Ward & - & - & - & - & 0.001 \\
\hline Forensic psychiatry & 25 & 32.9 & 31 & 63.3 & - \\
\hline Mentally disabled patients & 29 & 38.2 & 14 & 28.6 & - \\
\hline \multirow[t]{2}{*}{ Orthopsychiatry } & 22 & 28.9 & 4 & 8.2 & - \\
\hline & 54 & 71.1 & 43 & 87.8 & - \\
\hline Male sex & - & - & - & - & 0.03 \\
\hline Mean duration of follow-up (days) & 181.4 & - & 157.1 & - & 0.28 \\
\hline Involuntary admission (mean percentage of total follow-up) & $41 \%$ & - & $42 \%$ & - & 0.92 \\
\hline \multicolumn{6}{|l|}{ Diagnosis at hospital admission (DSM IV) } \\
\hline \multicolumn{6}{|l|}{ Axis I } \\
\hline Psychotic disorder & 37 & 48.7 & 28 & 57.1 & 0.34 \\
\hline Schizophrenia & 33 & 43.4 & 23 & 46.9 & 0.70 \\
\hline Alcohol dependence/abuse & 6 & 7.9 & 7 & 14.3 & 0.25 \\
\hline Drug dependence/abuse & 18 & 23.7 & 13 & 26.5 & 0.72 \\
\hline Mood disorder & 5 & 6.6 & 4 & 8.2 & 0.74 \\
\hline Development disorder & 11 & 14.5 & 0 & 0 & 0.01 \\
\hline More than one Axis I diagnosis & 22 & 28.9 & 14 & 28.6 & 0.96 \\
\hline \multicolumn{6}{|l|}{ Axis II } \\
\hline Personality disorder & 12 & 15.8 & 13 & 26.5 & 0.14 \\
\hline Cluster B & 9 & 11.8 & 6 & 12.2 & 0.95 \\
\hline Mental retardation & 30 & 39.5 & 13 & 26.5 & 0.14 \\
\hline \multicolumn{6}{|l|}{ Regular medication users at inclusion } \\
\hline Antipsychotics & 37 & 48.7 & 30 & 61.2 & 0.17 \\
\hline Antidepressants & 15 & 19.7 & 8 & 16.3 & 0.63 \\
\hline Anticonvulsants & 6 & 7.9 & 3 & 6.1 & 0.71 \\
\hline Benzodiazepines & 25 & 32.9 & 16 & 32.7 & 0.98 \\
\hline Promethazine & 2 & 2.6 & 2 & 4.1 & 0.65 \\
\hline Polypharmacy ${ }^{* *}$ & 53 & 69.7 & 34 & 69.4 & 0.97 \\
\hline Somatic medication & 23 & 30.3 & 18 & 36.7 & 0.45 \\
\hline
\end{tabular}

"The chi-squared test was used for categorical variables and the Mann-Whitney U test for the non parametric variables. "'More than one regular psychotropic drug prescribed.

Table 2 Characteristics of the aggressive incidents

\begin{tabular}{|c|c|c|c|c|c|c|}
\hline \multirow[t]{2}{*}{ Ward } & \multicolumn{2}{|c|}{ Incidents per bed per year } & \multicolumn{2}{|l|}{ Score* } & \multirow{2}{*}{$\begin{array}{l}\text { Only verbal } \\
\text { aggression } \\
(\%)\end{array}$} & \multirow{2}{*}{$\begin{array}{l}\text { Victim of the aggressive } \\
\text { incident needs treatment } \\
(\%)\end{array}$} \\
\hline & Mean & SD & Mean & SD & & \\
\hline Forensic psychiatry & 6.0 & 0.4 & 9.2 & 4.6 & 42.3 & 1.2 \\
\hline Mentally disabled patients & 10.6 & 0.7 & 11.0 & 4.3 & 31.6 & 4.2 \\
\hline Orthopsychiatry & 14.7 & 1.2 & 6.9 & 4.0 & 42.0 & 1.1 \\
\hline Total & 9.1 & 0.4 & 9.1 & 4.6 & 37.8 & 2.3 \\
\hline
\end{tabular}

"SOAS-R scores range from 0 to 22.

(96.1 \%) - predominantly oxazepam, diazepam, and temazepam - followed by promethazine (3.0\%), and antipsychotics (0.9\%). Psychotropic as-needed medication was most frequently administered orally (96.1\%). In the time-window of 48 hours around the aggressive incidents, $15 \%$ of the psychotropic as-needed medication was administered.

Main reasons for administration of psychotropics were comprised of "patient's demand" (53\%), distress (16.6\%), and sleep (13.7\%) for non-aggressive patients, and distress (36.6\%), sleep (17.3\%), patient's demand (16.6\%), and restlessness (13.1\%) for aggressive patients.

Acetaminophen was the most frequently administered somatic as-needed medication (72.2\%), followed by ibuprofen (16.1\%). Main reasons for administration of somatic as-needed medication were comprised of headache (33.3\%), "other somatic problems" (23.7\%), pain other than headache (22.1\%), and patient's demand (14.9\%) for non-aggressive patients, and headache (45.9\%), pain other than headache (39.8\%), and patient's demand (5.4\%) for aggressive patients.

Use of as-needed medication in aggressive vs. nonaggressive patients

The ID for the use of psychotropic as-needed medication in aggressive patients was 5.2 administrations per person-month compared to 2.1 administrations per person-month for nonaggressive patients, corresponding to an IDR of 2.4 (95\% confidence interval $[\mathrm{CI}] 2.2-2.7)$. In the multiplicative intensity model these results appeared to be valid. For oxazepam, which was administered most frequently to both aggressive and nonaggressive patients, the highest IDR was observed (IDR, 6.9; 95\% CI 5.6-8.5). 
Table 3 The use of as-needed medication of non-aggressive, mildly and severely aggressive patients

\begin{tabular}{|c|c|c|c|c|c|c|c|c|c|c|c|c|c|}
\hline \multirow[t]{3}{*}{$\begin{array}{l}\text { As-needed } \\
\text { medication }\end{array}$} & \multicolumn{3}{|c|}{$\begin{array}{l}\text { Patients without aggressive } \\
\text { incident(s) }(n=49)\end{array}$} & \multicolumn{5}{|c|}{$\begin{array}{l}\text { Patients with mild aggressive } \\
\text { incident(s) }(n=15)\end{array}$} & \multicolumn{5}{|c|}{$\begin{array}{l}\text { Patients with severe aggressive } \\
\text { incident(s) }(n=61)\end{array}$} \\
\hline & \multicolumn{2}{|c|}{ Users } & \multirow[t]{2}{*}{ ID $^{*}$} & \multicolumn{2}{|c|}{ Users } & \multirow[t]{2}{*}{$\mathrm{ID}^{*}$} & \multirow[t]{2}{*}{ IDR $^{* *}$} & \multirow[t]{2}{*}{$95 \% \mathrm{Cl}$} & \multicolumn{2}{|c|}{ Users } & \multirow[t]{2}{*}{ ID $^{*}$} & \multirow[t]{2}{*}{ IDR $^{* *}$} & \multirow[t]{2}{*}{$95 \% \mathrm{Cl}$} \\
\hline & $\mathbf{n}$ & $\%$ & & $\mathbf{n}$ & $\%$ & & & & $\mathbf{n}$ & $\%$ & & & \\
\hline Psychotropic & 20 & 41 & 2.1 & 7 & 47 & 3.3 & 1.6 & $1.4-1.8$ & 37 & 61 & 5.8 & 2.7 & $2.5-3.0$ \\
\hline Oxazepam & 13 & 27 & 0.4 & 4 & 27 & 1.1 & 2.9 & $2.2-3.8$ & 26 & 43 & 3.0 & 8.1 & $6.6-10.0$ \\
\hline Diazepam & 5 & 10 & 0.9 & 1 & 7 & 1.2 & 1.4 & $1.1-1.7$ & 10 & 16 & 1.2 & 1.3 & $1.1-1.6$ \\
\hline Temazepam & 10 & 20 & 0.6 & 4 & 27 & 0.6 & 0.9 & $0.7-1.3$ & 11 & 18 & 0.8 & 1.2 & $1.0-1.5$ \\
\hline Other & 4 & 8 & 0.2 & 3 & 20 & 0.4 & 1.7 & $1.1-2.6$ & 15 & 25 & 0.8 & 3.6 & $2.7-4.8$ \\
\hline Somatic & 22 & 45 & 1.0 & 13 & 87 & 1.6 & 1.7 & $1.4-2.0$ & 43 & 70 & 2.2 & 2.2 & $1.9-2.5$ \\
\hline
\end{tabular}

"Incidence density per person-month. ${ }^{* *}$ Incidence density ratio; reference-group is the group of patients without aggressive incidents.

The same analysis was performed for the use of somatic asneeded medication, resulting in IDs for aggressive patients and non-aggressive patients of 2.1 and 1.0 per person-month, respectively. The IDR was 2.1 (95\% CI 1.8-2.4). The observed increased use of as-needed medication for aggressive patients was higher for severely aggressive patients compared to mildly aggressive patients, except for diazepam. (o Table 3).

Stratified analyses for the three different wards for psychotropics and somatics as-needed medication revealed statistically significant increased use of as-needed medication for the aggressive patients in all three wards.

\section{Time relationship between as-needed medication and aggressive incidents}

During the nine-month study period, 551 outward directed aggressive incidents were recorded. For $75 \%$ of the registered aggressive incidents, no as-needed medication was administered during the 48-hours time-window around the aggressive incident. For most incidents, the number of administrations before an aggressive incident equaled the number of administrations following an aggressive incident (no difference of use at $3 \mathrm{~h}: 86 \%, 12 \mathrm{~h}: 83 \%, 24 \mathrm{~h}: 82 \%, 48 \mathrm{~h}: 78 \%)$. Intensity of use of psychotropic as-needed medication per 3 hours periods within the 24 hours time window before and following an aggressive incident was calculated and displayed in 0 Fig. 1. The highest intensity of as-needed medication use was observed in the three hours following an aggressive incident. Comparing the intensity of use in this period with the intensity of use in the six hours before resulted in a ratio of 3.0 (95\% CI 1.3-6.8). More as-needed medications were administered in the 36 hours following an aggressive incident compared to the 36 hours before (intensity ratio [IR] $1.4 ; 95 \% \mathrm{CI} 1.1-1.8$ ). Furthermore, the intensity of use in the three-hour period following an aggressive incident was compared with the period from 36 hours to three hours before an aggressive incident and on the other hand with the period from three hours to 36 hours following aggressive incidents (IR 3.9; 95\% CI 2.0-7.7 and IR 1.84; 95\% CI 1.2-2.9, respectively).

\section{Discussion}

$\nabla$

In this observational study, conducted in three Dutch long stay wards, the association between the use of as-needed medication and aggressive behavior was investigated. In line with previous findings that aggressive patients have an increased use of psy-

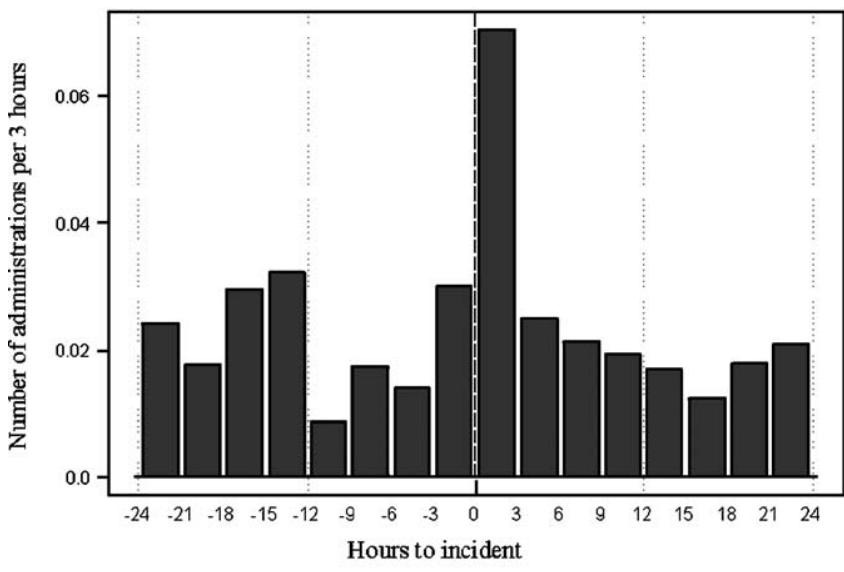

The thick line indicates the occurrence of an aggressive incident $(\mathrm{t}=0)$.

Fig. 1 Intensity of as-needed medication use around aggressive incidents

chotropics - higher dosages and more polypharmacy $[9,10]$ - an increased use of psychotropic as-needed medication was observed. Interestingly, in addition to an increased use of psychotropic as-needed medication the use of somatic as-needed medication was also found to be increased. Apparently, aggressive behavior triggers the use of as-needed medication.

Strengths of our study include the use of a validated aggression measurement instrument (the SOAS-R) and the actual use of asneeded medication as study outcome instead of the prescription of as-needed medication. Previous studies either assessed the reasons for prescription or administration of as-needed medication without the use of an aggression measurement instrument and without a group of non-aggressive patients as reference group $[2,3,6]$. To our knowledge, only Soliman and Reza compared non-aggressive patients to aggressive patients [9]. However, in that study, not the use of as-needed medication was analyzed, but the prescription of as-needed medication. Furthermore, in the study of Soliman and Reza, aggression was retrospectively assessed from hospital records by the researchers with the use of the SOAS-R, although the SOAS-R is designed to record aggression as soon as possible after the aggressive incident by the staff witnessing the incident.

Looking at the temporal associations between episodes of aggressive incidents and the administration of as-needed medication immediately preceding and following these aggressive incidents, an increased intensity of use was observed shortly 
after the aggressive incident. It seems fair to conclude that in those cases as-needed medication is used to regain control and/ or prevent further escalation. From a pharmacological point of view, the choice for oxazepam seems irrational, as the optimal effect of oxazepam is only reached after two to three hours. A better choice, to our point of view, would be lorazepam or clorazepate, which have a shorter $\mathrm{T}_{\max }$.

The interval between the administration of as-needed medication and an aggressive incident was frequently much larger than 48 hours, which suggests that aggressive behavior leads to the administration of as-needed medication, not only immediately following aggressive incidents but also on other moments. This suggestion was reinforced by the observation that the intensity of use in the time-window of three to 36 hours following the aggressive incident was increased compared to the intensity of use in the time window of three to 36 hours before an aggressive incident. We, therefore, hypothesized that as-needed medication is not only used for the (direct) management of aggression. This hypothesis was further strengthened by the observation that not only the use of psychotropic as needed medications was increased, but also the use of somatic as-needed medications. One could think of different explanations, which could not be investigated with the available data. A plausible reason for an increased use of as-needed medication might be that the staff tends to administrate more as-needed medication to patients, once they have shown aggressive behavior, in order to prevent future aggressive incidents. However, it might also well be possible that aggressive patients are keener on as-needed medication than non-aggressive patients and make a stronger appeal on the staff to obtain as-needed medication. Still another explanation might be that aggressive patients are more severely ill, thereby needing more medication, e.g., for insomnia.

Limitations of this study are the following. Firstly, due to the observational study-design, an association between aggression and as-needed medication could be determined, but we could not investigate the causality of this observation, i.e., "is the increased use of as-needed medication caused by aggression itself or by other determinants associates with aggression?"; only hypotheses could be formulated.

Secondly, for the assessment of aggression, only the SOAS-R was used. By measuring aggression with the SOAS-R, we had to rely on staff's willingness to report witnessed aggressive behavior. Such measurement probably resulted in a certain degree of underreporting, especially in the case of mildly aggressive behavior. The degree of underreporting was not assessed. However, the incidence of aggressive incidents was higher than the median incidence observed in the review of Nijman et al [8]., in which studies with aggression data, measured by the SOAS-R, were analyzed.

Furthermore, reporter-bias could have been occurred as the staff, who registered both the administration of medication and aggression, could be more willing to report an aggressive incident after administering as-needed medication. On the other hand, as the registration of as-needed medication is in practice for many years and as around many aggressive incidents no as-needed medication was administered, the risk of such bias seems small. Lastly, the heterogeneity of the study population might have resulted in a biased risk estimation. With a dynamic study population, both chronically and newly admitted patients were included in this study, which resulted in a heterogeneous study population. With the participation of three different wards, the heterogeneity of the study population was further increased. Some heterogeneity of these three wards was reflected in the differences in severity and frequency of the aggressive incidents. Mean incidence and the percentage of victims requiring treatment, however, all fell in the range observed in the review [8]. It, therefore, seemed reasonable to analyze the data of the three wards together instead of conducting stratified analyses, which would have decreased significantly the statistical power.

Taking into account our study limitations, we conclude that aggressive patients use more as-needed medication than nonaggressive patients. However, to a certain extent, the function of as-needed medication practices is unclear. As-needed medication administered immediately preceding or following the occurrence of aggressive incidents, is mainly administered to regain control and/or prevent further escalation. For the other as-needed medications administrations we can only make guesses about their function and effectiveness. A tempting hypothesis is that aggressive patients are keen on as-needed medication, which they might obtain by showing demanding behavior. However, on the basis of this study it also might be possible that aggressive behavior in the past leads to the administration of as-needed medication in the future, with the aim of preventing the occurrence of other aggressive incidents.

\section{Acknowledgment}

$\nabla$

We gratefully acknowledge the contribution of Svetlana V. Belitser to the data-analysis.

\section{References}

1 Andersen PK, Borgan $O$ et al: Statistical models based on counting processes. New York: Springer, 1997

2 Craig TJ, Bracken J: An epidemiological study of prn/stat medication use in a state psychiatric hospital. Ann Clin Psychiatry 1995; 7 (2): 57-64

3 Craven JL, Voore PM et al: PRN medication for psychiatric inpatients. Can J Psychiatry 1987; 32 (3): 199-203

4 Goedhard LE, Stolker JJ et al: Pharmacotherapy for the treatment of aggressive behavior in general adult psychiatry: A systematic review. J Clin Psychiatry 2006; 67 (7): 1013-1024

5 Hunter $M$, Carmel $H$ : The cost of staff injuries from inpatient violence. Hosp Community Psychiatry 1992; 43: 586-588

6 McLaren S, Browne FW et al: A study of psychotropic medication given 'as required' in a regional secure unit. Br J Psychiatry 1990; 156: 732-735

7 Nijman H, Muris P et al: The staff observation aggression scale-Revised (SOAS-R). Aggress Behav 1999; 25: 197-209

8 Nijman HL, Palmstierna T et al: Fifteen years of research with the Staff Observation Aggression Scale: a review. Acta Psychiatr Scand 2005 111 (1): $12-21$

9 Soliman $A E$, Reza H: Risk factors and correlates of violence among acutely ill adult psychiatric inpatients. Psychiatr Serv 2001; 52 (1): 75-80

10 Stolker IJ, Heerdink ER et al: Determinants of multiple psychotropic drug use in patients with mild intellectual disabilities or borderline intellectual functioning and psychiatric or behavioral disorders. Gen Hosp Psychiatry 2001; 23 (6): 345-349

11 Thapa PB, Palmer SL et al: P. R.N. (As-needed) orders and exposure of psychiatric inpatients to unnecessary psychotropic medications. Psychiatr Serv 2003; 54 (9): 1282-1286

12 Whicher E, Morrison $M$ et al: As required' medication regimens for seriously mentally ill people in hospital. Cochrane Database Syst $\operatorname{Rev}(2)$ 2002;CD003441 